\title{
Impact of the Covid-19 pandemic on food aid: a qualitative exploration
}

\author{
H. Mulrooney ${ }^{1}$, D. Bhakta ${ }^{2}$ and R. Ranta ${ }^{3}$ \\ ${ }^{1}$ School of Life Sciences, Pharmacy and Chemistry, Kingston University, London, UK, \\ ${ }^{2}$ School of Human Sciences, London Metropolitan University, London, UK and \\ ${ }^{3}$ School of Law, Social and Behavioural Sciences, Kingston University, London, UK
}

The Covid-19 pandemic resulted in a well-documented increase in the demand for food aid in the $\mathrm{UK}^{(1,2)}$ driven by difficulties in accessibility, availability, utilisation and stability, the four pillars of food insecurity ${ }^{(3)}$. In response, community food aid was scaled up at an unprecedented speed and scale. The innovations which enabled this and how sustainable they are for future provision, are unclear.

This project aimed to explore food aid provision in South West London and Sussex through the lens of those involved in its provision. Specific objectives included:

- Identifying changes which occurred in response to the pandemic;

- Exploring the impact of such changes on food aid provision;

- Developing an understanding of the sustainability of such changes.

Ethical approval for the project was obtained from Kingston University Research Ethics Committee. Semi-structured audiorecorded interviews were carried out online with workers and volunteers at pre- existing and new food aid organisations. These were identified initially using researcher knowledge (one is a food aid volunteer), and desk-based research. Subsequently, interviewees identified other contacts using a snowballing technique. An interview guide was used for consistency; interviews typically lasted $30-45$ minutes. Interviews aimed to explore experience of food aid prior to and during the pandemic, the impact of the pandemic, the meaning of food aid involvement for the individuals and their views on sustainability of changes which had occurred. When restrictions were lifted, a number of site visits were also undertaken. Transcripts were analysed using basic thematic analysis.

A total of 27 interviews and 6 site visits have been undertaken to date in this ongoing project. A number of major themes were identified. Increased need for support occurred resulting from difficulties with all four pillars of food insecurity; availability (food shortages), access (difficulty obtaining online shopping slots), utilisation (physical ill-health) and stability (altered employment and income). Innovations occurred between organisations to meet this need including enhanced cooperation, communication and sharing of food and resources. Altered organisational practices to accommodate different demands were reported (e.g. food deliveries in place of collections). A more holistic response seeking to address underlying issues (e.g. development of a structured local response over time), signposting to appropriate help and support (e.g. benefits support) and enhanced skills development (e.g. provision of recipe boxes), was also found. The extent to which improvements identified are sustainable in the longer term is unclear.

Many positive innovations in response to the pandemic were identified. Their long-term sustainability is unclear, as is the extent to which support will be maintained once the crisis is perceived to be over. Addressing the drivers of food insecurity is critical to help drive down future need for emergency food support.

\section{Acknowledgments}

This work was supported by a CresCID grant from Kingston University.

\section{References}

1. Trussell Trust (2021) [Available at: https://www.trusselltrust.org/wp-content/uploads/sites/2/2021/05/State-of-Hunger-2021-Report-Final.pdf].

2. Macaninch E, Martyn K \& Lima do Vale M (2020) BMJ Nutrition, Prevention \& Health.

3. FAO (2006) [Available at: http://www.fao.org/fileadmin/templates/faoitaly/documents/pdf/pdf_Food_Security_Cocept_Note.pdf]. 